\title{
Ipr1 modified BCG as a novel vaccine induces stronger immunity than BCG against tuberculosis infection in mice
}

\author{
YUWEI WANG ${ }^{1}$, CHUN YANG $^{2,3}$, YONGLIN HE ${ }^{2,3}$, XINGXING ZHAN $^{4}$ and LEI XU ${ }^{2,3}$ \\ ${ }^{1}$ Department of Laboratory Medicine, Chongqing Hospital of Traditional Chinese Medicine, Chongqing 400021; \\ ${ }^{2}$ Department of Microbiology; ${ }^{3}$ Department of Molecular Medicine and Cancer Research Center; \\ ${ }^{4}$ Department of Pediatrics, Chongqing Medical University, Chongqing 400016, P.R. China
}

Received March 16, 2015; Accepted March 18, 2016

DOI: $10.3892 / \mathrm{mmr} .2016 .5447$

\begin{abstract}
Tuberculosis is a major challenge to global public health. However, the Bacille Calmette-Guérin (BCG), the only vaccine available against tuberculosis, has been questioned for the low protective effect. The present study used the mouse gene intracellular pathogen resistance I (Iprl) gene to alter the current BCG vaccine and evaluated its immunity effect against tuberculosis. This study also investigated the intrinsic relationships of Iprl and innate immunity. The reformed BCG (BCGi) carrying the Iprl gene was constructed. The mice were intranasally challenged with the M.tuberculosis H37Rv strain after vaccination with BCGi. Protection efficacy of the vaccine was assessed by the organ coefficient, bacterial load and pathological changes in the lung. The differential expression of 113 immune-related genes between BCGi and BCG groups were detected by an oligo microarray. According to the results of organ coefficient, bacterial load and pathological changes in the organization, BCGi had been shown to have stronger protective effects against $M$. tuberculosis than BCG. The oligo microarray and reverse transcription-quantitative polymerase chain reaction further revealed that the Iprl gene could upregulate the expression of 13 genes, including a $>3$-fold increase in Toll-like receptor (TLR) 4 and 10-fold increase in surfactant protein $\mathrm{D}(s f t p d)$. The two genes not only participate in innate immunity against pathogens, but also are closely interrelated. Iprl could activate the TLR4 and sftpd signaling pathway and improve the innate immunity against tuberculosis, therefore Iprl modified BCG may be a candidate vaccine against M. tuberculosis.
\end{abstract}

Correspondence to: Dr Lei Xu, Department of Microbiology, Chongqing Medical University, 1 Yixueyuan Road, Yuzhong, Chongqing 400016, P.R. China

E-mail: bear111xu@126.com

Key words: Bacille Calmette-Guérin, intracellular pathogen resistance I, tuberculosis, Toll-like receptors, surfactant protein D

\section{Introduction}

Tuberculosis (TB) is an infectious disease and remains one of the leading infectious causes of fatality worldwide. Factors including co-infection with HIV, extending the treatment (at least 6 months) and the emergence of drug resistant strains put more burdens on the treatment of TB $(1,2)$. One of the World Health Organization (WHO) Millennium Development Goals is to reduce tuberculosis deaths by $90 \%$ and to reduce the number of new cases by $80 \%$ between 2015 and 2030 . However, >8.5 million tuberculosis cases were reported in 2011, with an increase in the number of multidrug-resistant strains (3). Therefore, the WHO target cannot be reached without the development of a vaccine to limit the spread of TB.

Currently, Bacille Calmette-Guérin (BCG) is the only vaccine available against TB and it has been questioned for the low protective effect against TB in adults and adolescents $(3,4)$. Therefore, a new vaccine or an alteration of the current BCG vaccine is urgently required in order to promote cellular immune responses against Mycobacterium tuberculosis (M. tuberculosis).

In 2005, Pan et al (5) found a gene in C57BL/6J mice that was able to promote macrophage resistance to intracellular pathogens and was designated intracellular pathogen resistance I (Iprl). Iprl has been shown to promote the activation of macrophages against M.tuberculosis infection in vitro (5). However the mechanisms remained unclear.

In the present study, a recombinant BCG carrying IprI (BCGi) was constructed and its immune effect against M. tuberculosis was examined in vivo. Then, the oligo microarray assay was conducted to further investigate the mechanisms underlying the effect of Iprl on the prevention of M. tuberculosis infection.

\section{Materials and methods}

Construction of BCGi. Coding sequences of Iprl, green fluorescent protein (GFP) and OriM (mycobacterial origin of replication) were simultaneously cloned into pBudCE4.1, which has multiple promoters to generate the shuttle plasmid pBudCE4.1/Ipr1/GFP/OriM (pBGOI). The pBGOI was 
transformed into BCG. The recombinant BCG carrying Iprl (BCGi) had been successfully constructed previously (6).

Bacterial strains, media and growth conditions. BCG Pasteur (2003050401) was obtained from the Shanghai Institute of Biological Products (Shanghai, China). The M. tuberculosis H37Rv strain was obtained from the Microbiology Biosafety Laboratory of Tongji Medical College of HUST, Huazhong University (Wuhan, China). BCG, BCGi and H37Rv were routinely cultured in Middlebrook 7H9 (Difco; BD Biosciences, Franklin Lakes, NJ, USA) broth containing 10\% OADC, $0.2 \%$ (v/v) glycerol, $0.05 \%$ (v/v) Tween 80 (all Difco) and Löwenstein-Jensen media (produced internally, containing: Malachite green, glycerol, asparagine, potato starch, coagulated eggs, potassium dihydrogen phosphate,magnesium sulfate and sodium citrate) for 4 weeks. They were then harvested and diluted to $5 \times 10^{5}$ colony-forming units (cfus) $/ 50 \mu \mathrm{l}$, in saline containing $0.05 \%$ Tween 80 .

Animals. Specific pathogen-free (SPF) 6-8 week old female $\mathrm{C} 3 \mathrm{HeB} / \mathrm{FeJ}$ mice were obtained from the Model Animal Research Institute of Nanjing University (Nanjing, China). Mice were kept in specific pathogen-free housing in a temperature/humidity-controlled environment $\left(\sim 24^{\circ} \mathrm{C}\right)$, with a 12-h dark:light cycle and free access to food and water in the Biosafety Laboratory of Tongji Medical College, Huazhong University (Wuhan, China). The experimental protocols complied with the ethics guidelines of Chongqing Medical University and Huazhong University, China.

Intranasal vaccination with BCGi in vivo. The mice were divided into 3 groups (control group, BCG group, BCGi group) at random, with 10 mice in each group and another five normal mice were used as controls. Once anesthetized for $10 \mathrm{sec}$ with ether, BCGi was administered to mice intranasally. The BCGi group received $\mathrm{BCGi}\left(5 \times 10^{5} \mathrm{cfu} / 50 \mu \mathrm{l}\right)$ a total of 7 times at 3-day intervals over 3 weeks. The BCG group received BCG $\left(5 \times 10^{5} \mathrm{cfu} / 50 \mu \mathrm{l}\right)$ and the control group received $50 \mu \mathrm{l}$ saline.

Animal challenge study. The experiment started 4 weeks after vaccination and mice were intranasally challenged with the live H37Rv $\left(5 \times 10^{5} \mathrm{cfu} / 50 \mu \mathrm{l}\right)$. Successful infection was confirmed by the bacterial counts of 2 mice sacrificed by cervical dislocation 3 days after infection. Control mice received $50 \mu \mathrm{l}$ sterile $0.9 \% \mathrm{NaCl}$ solution only. The experimental mice were sacrificed on the 11th week after the first vaccination.

Immunization efficacy. Protection efficacy of the vaccine against $M$. tuberculosis was assessed as follows: i) Iprl expression in lung tissues; ii) organ coefficient; iii) M. tuberculosis load in the lungs and spleen; and iv) histopathological characterization of the infected organs.

Iprl expression in organs. Firstly, the fresh lung and spleen tissues were lysed by TRIzol (Takara Biotechnology Co., Ltd., Dalian, China) for $5 \mathrm{~min}$ at room temperature. Secondly, samples were lysed at $10,000 \mathrm{x}$ g for $10 \mathrm{~min}$, and the pellet was discarded. Chloroform was added and the lysate was placed on ice for $5 \mathrm{~min}$ after mixing upside down, and then centrifuged at $16,000 \mathrm{x}$ g under $4^{\circ} \mathrm{C}$ for $15 \mathrm{~min}$. The upper aqueous phase was transferred to another centrifuge tube and placed on ice for 10 min after mixing with isopropanol. RNA sunk to the bottom of the tube following centrifugation at $10,000 \mathrm{x} \mathrm{g}$ for $10 \mathrm{~min}$ at $<4^{\circ} \mathrm{C}$. Ethanol was added to the centrifuge tube, and the precipitate was suspended with gentle shaking followed by centrifugation at $4^{\circ} \mathrm{C}$ at $8,000 \mathrm{x} \mathrm{g}$ for $5 \mathrm{~min}$ in order to discard the supernatant. Once dried at room temperature, using $20 \mu \mathrm{l}$ DEPC treated water (Takara Biotechnology Co., Ltd.) to dissolve the RNA sample. Ipr1 was detected by reverse transcription-polymerase chain reaction. Total RNA was extracted from fresh lung and spleen tissues. Total RNA $(1 \mu \mathrm{g})$ was used for cDNA synthesis, which was conducted by reverse transcription using the PrimeScript RT reagent kit Perfect Real time (Takara Biotechnology Co., Ltd.). PCR the Iprl gene with the primers (shown in Table I), with $\beta$-actin mRNA as an endogenous control. It was also examined by western blot analysis. The proteins were extracted using RIPA buffer (Beyotime Institute of Biotechnology, Shanghai, China) supplemented with PMSF (Beyotime Institute of Biotechnology). Protein concentrations were determined using a BCA protein concentration determination kit (Beyotime Institute of Biotechnology). Equal amounts of sample were separated using $10 \%$ sodium dodecyl sulphate-polyacrylamide gel electrophoresis (SDS-PAGE; Beyotime Institute of Biotechnology) and transferred onto polyvinylidene fluoride membranes (PVDF; Takara Biotechnology Co., Ltd.). The PVDF membranes were blocked with 5\% skimmed milk. Subsequent to incubation with polyclonal rabbit anti-Ipr1 antibody (cat.no. 4407; ProSci, Poway, CA, USA; diluted 1:250) for $12 \mathrm{~h}$, the PVDF membrane was blocked with skimmed milk after washing three times with TBST buffer. Following this, membranes were incubated with the monoclonal goat anti-rabbit-IgG antibody (cat. no. sc-2004; Santa Cruz Biotechnology, Inc., Dallas, TX, USA; diluted 1:1,000) for $1 \mathrm{~h}$. After washing three times with TBST buffer, signals were detected using the DAB Horseradish Peroxidase Color Development kit (Beyotime institute of Biotechnology). Cells in which cytoplasm stained brown-yellow were considered positive.

Coefficient of lung and spleen. The weight of each mouse was taken at 11 weeks after the initial vaccination. The lungs and spleens were immediately removed after the mice were sacrificed. The weight of each lung and spleen was measured an organ coefficients were determined using the following formula: Organ coefficient $=$ organ weight $(\mathrm{g}) /$ mouse weight (g) x 100 .

M. tuberculosis load in lung and spleen. The entire left lobe of the lung and one third of the spleen was weighed accurately and aseptically. These organs were individually homogenized in sterile saline containing $0.05 \%$ Tween 80 . Ten-fold serial dilutions of the homogenates were placed onto Löwenstein-Jensen media containing $2 \mathrm{mg} / \mathrm{l} \mathrm{BCG}$ inhibitor TCH (2-thiophenecarboxylic acid hydrazide; Difco). Bacterial counts in organs were counted and shown in $\log 10 \mathrm{cfu} / \mathrm{g}$ after incubation at $37^{\circ} \mathrm{C}$ for 4 weeks.

Immunohistochemistry. The experimental mice were sacrificed at the 11th week after the first vaccination. Approximately half of the right lobe of the lung and one third of the spleen were immersed in $4 \%$ paraformaldehyde for $4 \mathrm{~h}$, and transfered to $70 \%$ ethanol. Individual lobes of lung and spleen tissue biopsy material was placed in processing cassettes, 
Table I. Primers used for reverse transcription-quantitative polymerase chain reaction.

\begin{tabular}{|c|c|c|c|c|}
\hline Symbol & Gene ID & Primers & $\begin{array}{l}\text { PCR product } \\
\text { length (bp) }\end{array}$ & $\operatorname{Tm}\left({ }^{\circ} \mathrm{C}\right)$ \\
\hline Gapdh & NM_008084.2 & $\begin{array}{l}\text { F: 5'-GTTGTCTCCTGCGACTTCA-3' } \\
\text { R: 5'-GCCCCTCCTGTTATTATGG-3' }\end{array}$ & 293 & 59 \\
\hline Igh-6 & XM_177464 & $\begin{array}{l}\text { F: 5'-GGTGCACAGAAATCAAACCC-3' } \\
\text { R: 5'-CCAGGAGCTACTGAATGCGT-3' }\end{array}$ & 240 & 59 \\
\hline$L b p$ & NM_008489 & $\begin{array}{l}\text { F: 5'-TCACCGCTCTCCAGTTGCTA-3' } \\
\text { R: 5'-GATGCCGGAGTCATGTGGTA-3' }\end{array}$ & 177 & 59 \\
\hline$L t f$ & NM_008522 & $\begin{array}{l}\text { F: 5'-TCAATGGTGTGCTGTGTC-3' } \\
\text { R: 5'-CTGCTACCGCATAGTAGTGA-3' }\end{array}$ & 211 & 59 \\
\hline Ly96 & NM_016923 & $\begin{array}{l}\text { F: 5'-CTCTTTTCGACGCTGCTTTC-3' } \\
\text { R: 5'-TTCCTTACGCTTCGGCAACT-3' }\end{array}$ & 260 & 59 \\
\hline$N c f 4$ & NM_008677 & $\begin{array}{l}\text { F: 5'-CACCAACTGGCTACGATGCT-3' } \\
\text { R: 5'-CATCCTCATCTGACAGCAGC-3' }\end{array}$ & 198 & 59 \\
\hline Nfkbl & NM_008689 & $\begin{array}{l}\text { F: 5'-GGCCCATACCTTCAAATATTAGAG-3' } \\
\text { R: 5'-GTGACCAACTGAACGATAACCTT-3' }\end{array}$ & 181 & 59 \\
\hline$N f \kappa b 2$ & NM_019408 & $\begin{array}{l}\text { F: 5'-TATGCCATTGTGTTCCGGAC-3' } \\
\text { R: 5'-TCCTCCTTGTCTTCCACCAG-3' }\end{array}$ & 148 & 59 \\
\hline$N f \kappa b i a$ & NM_010907 & $\begin{array}{l}\mathrm{F}: \text { 5'-ACGAGGAGTACGAGCAAATGG-3' } \\
\mathrm{R}: \text { 5'-CTCAGGATCACAGCCAGCTT-3' }^{\prime}\end{array}$ & 289 & 59 \\
\hline Nos 2 & NM_010927 & $\begin{array}{l}\text { F: 5'-AGACCCACATCTGGCAGAAT-3' } \\
\text { R: 5'-ACCAGCAGTAGTTGCTCCTCT-3' }\end{array}$ & 273 & 59 \\
\hline $\operatorname{Prg} 2$ & NM_008920 & $\begin{array}{l}\text { F: 5'-GAGAACTTGCCTAGGGATGC-3' } \\
\text { R: 5'-CTGACTAGGAGGTAGCGACAG-3' }\end{array}$ & 244 & 59 \\
\hline Sftpd & NM_009160 & $\begin{array}{l}\text { F: 5'-GGAGAACGTGGACTAAGTGGA-3' } \\
\text { R: 5'-TCTCCTTTAGGACCTGGTTTGC-3' }\end{array}$ & 121 & 59 \\
\hline Stabl & NM_138672 & $\begin{array}{l}\text { F: 5'-GTTCAGTCTGCCAGGAGTGC-3' } \\
\text { R: 5'-TCTTGCTGAGTGTATCCGGG-3' }\end{array}$ & 249 & 59 \\
\hline Tlr4 & NM_021297 & $\begin{array}{l}\text { F: 5'-TAGAGAATCTGGTGGCTGTG-3' } \\
\text { R: 5'-CCACATGTACTAGGTTCGTC-3' }\end{array}$ & 158 & 59 \\
\hline$C d 14$ & NM_009841 & $\begin{array}{l}\text { F: 5'-GGCTTGTTGCTGTTGCTTCT-3' } \\
\text { R: 5'-CGTGTCCACACGCTTTAGAA-3' }\end{array}$ & 197 & 59 \\
\hline$C d l d l$ & NM_007639 & $\begin{array}{l}\text { F: 5'-CGGTACCTACCATGGCTGTT-3' } \\
\text { R: 5'-CTGATGGTGGCTGAGTCATT-3' }\end{array}$ & 193 & 59 \\
\hline Chuk & NM_007700 & $\begin{array}{l}\text { F: 5'-GAACGTCAGTCTGTACCAGCA-3' } \\
\text { R: 5'-CCTCCAGAACAGTACTCCATTG-3' }\end{array}$ & 221 & 59 \\
\hline$C s f 3$ & NM_009971 & $\begin{array}{l}\text { F: 5'-GCACTATGGTCAGGACGAGAG-3' } \\
\text { R: 5'-CTGGCTTAGGCACTGTGTCTG-3' }\end{array}$ & 272 & 59 \\
\hline$P p b p$ & NM_023785 & $\begin{array}{l}\text { F: 5'-GCTTCAGACTCAGACCTACATC-3' } \\
\text { R: 5'-GGCTATCACTTCCACATCAG-3' }\end{array}$ & 250 & 59 \\
\hline Cxcr 4 & NM_009911 & $\begin{array}{l}\text { F: 5'-TGGAACCGATCAGTGTGAGT-3' } \\
\text { R: 5'-TACTTGTCCGTCATGCTCCT-3' }\end{array}$ & 231 & 59 \\
\hline Plunc & NM_011126 & $\begin{array}{l}\text { F: 5'-GAGCCTCGTTGTCCTCTGTG-3' } \\
\text { R: 5'-CACCGCTGAGAGCATCTGTG-3' }\end{array}$ & 232 & 59 \\
\hline
\end{tabular}

$\mathrm{R}$, reverse; $\mathrm{F}$, forward.

dehydrated through a serial alcohol gradient, and embedded in paraffin wax blocks. Prior to immunostaining, 5- $\mu$ m-thick lung/spleen tissue sections were dewaxed in xylene, rehydrated through decreasing concentrations of ethanol, and washed in PBS. Sections were then stained with hematoxylin and eosin. After staining, sections were dehydrated through increasing concentrations of ethanol and xylene. Using a biological microscope (OLYMPUS CX23; Olympus Corporation, Tokyo, Japan) to assess the pathological changes is the tissues.

Western blot analysis. Cells were washed three times by precooling PBS and add the lysis buffer which contain $1 \%$ Phosphatase inhibitors, $0.1 \%$ protease inhibitors and $0.5 \%$ PMSF. This was followed by pipetting, gently shaking at $4^{\circ} \mathrm{C}$ 

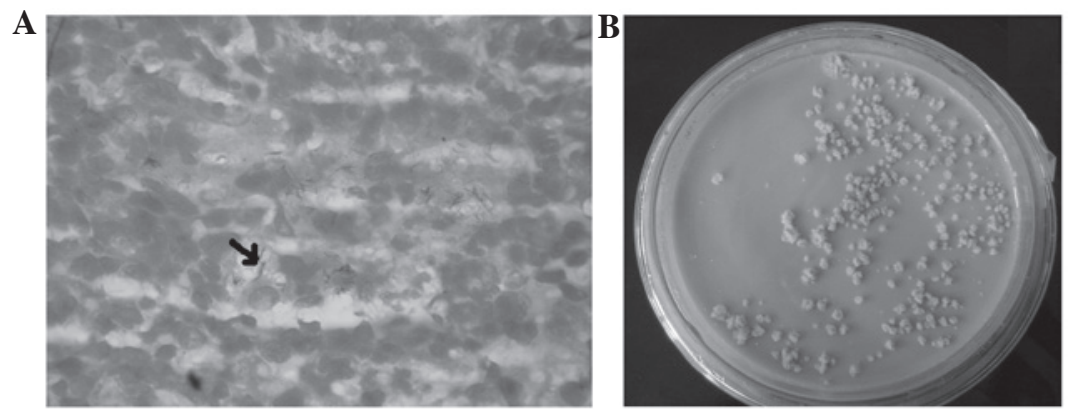

Figure 1. M. tuberculosis H37Rv strain was found in the lung tissues from the infected C3HeB/FeJ mice. (A) H37Rv in lung tissues by (magnification, $\mathrm{x} 1,000$ ). (B) H37Rv on Löwenstein-Jensen medium from the lung tissues of infected mice.
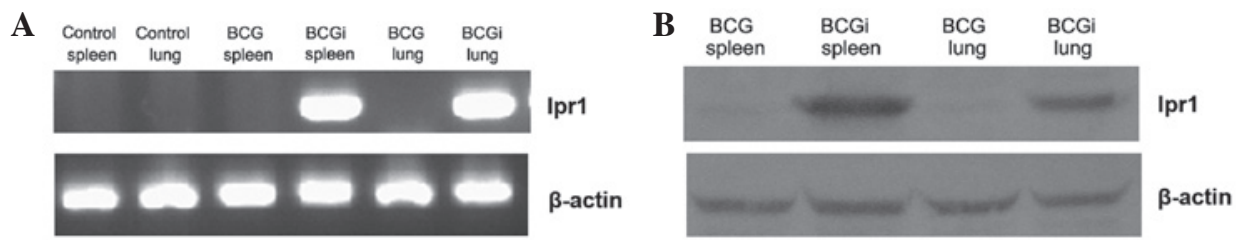

Figure 2. Ipr1 expression in lung and spleen tissues from BCGi vaccinated mice. (A) Ipr1 expression was detected by reverse transcription-quantitative polymerase chain reaction. (B) Ipr1 expression was examined by western blot analysis. Ipr1, intracellular pathogen resistance I; BCGi,Bacille Calmette-Guérin with Ipr1.
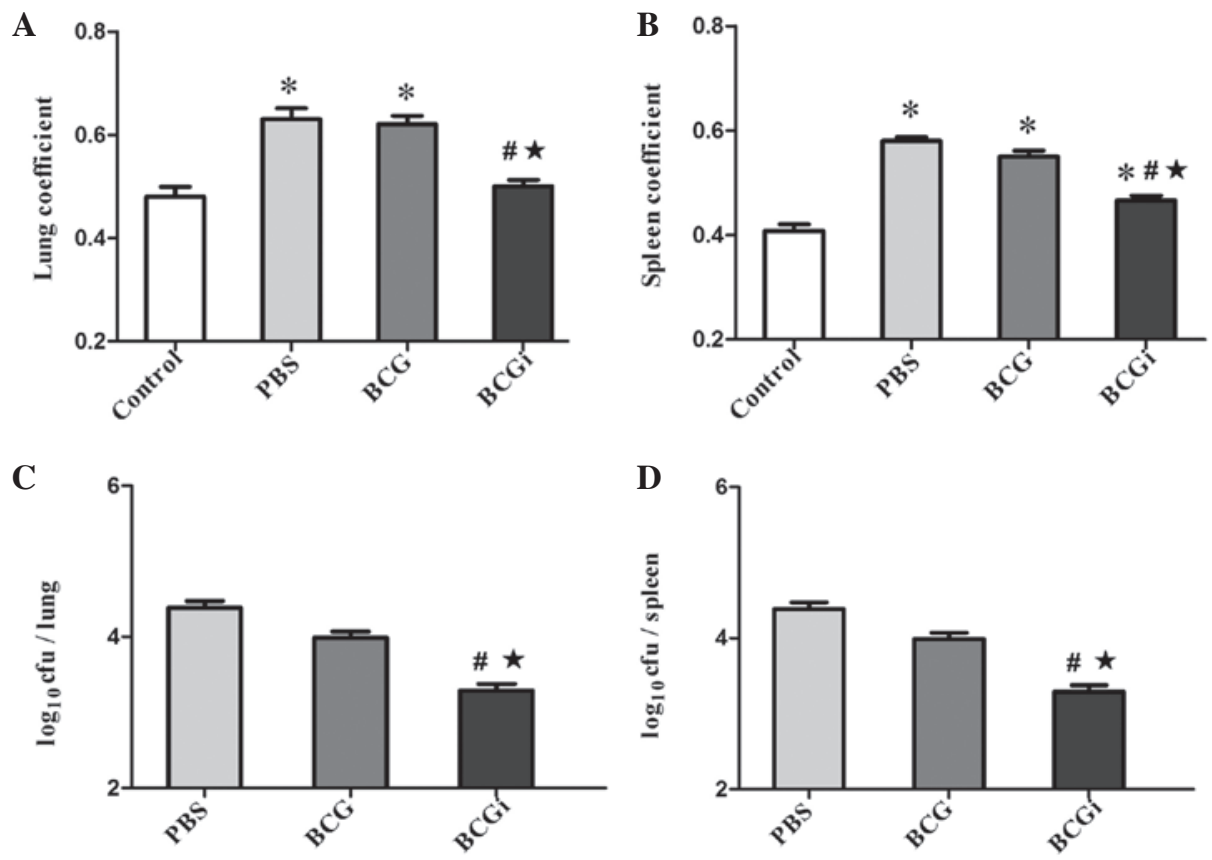

Figure 3. Organ coefficient and the bacterial load in lung and spleen tissues from the infected mice after vaccination. The $\mathrm{C} 3 \mathrm{HeB} / \mathrm{FeJ}$ mice were vaccinated with BCGi, BCG or PBS (as negative control) 7 times at 3-day intervals. Mice were intranasally challenged with the live H37Rv at 4 weeks after the last vaccination. The mice were sacrificed at another 4 weeks after H37Rv infection. Control group was vaccinated with PBS but not infected with H37Rv. (A) Lung coefficient. (B) Spleen coefficient. (C) Bacterium load in lung tissues. (D) Bacterium load in spleen tissues. ${ }^{*} \mathrm{P}<0.01$ vs. Control; ${ }^{*} \mathrm{P}<0.01 \mathrm{vs}$. PBS; ${ }^{\star} \mathrm{P}<0.01 \mathrm{vs}$. BCG. BCG, Bacille Calmette-Guérin; BCGi, BCG with intracellular pathogen resistance I; PBS, phosphate-buffered saline.

for $15 \mathrm{~min}$, then centrifugation at the speed of $12,000 \mathrm{x} \mathrm{g}$ at $4^{\circ} \mathrm{C}$. Protein concentrations were determined using a bicinchoninic acid protein concentration determination kit (Beyotime Institute of Biotechnology). Equal quantities of sample were separated using 10\% SDS-PAGE and transferred onto a PVDF membrane. The membrane was then blocked with $5 \%$ skimmed milk for $1 \mathrm{~h}$ at room temperature. After incubation with mouse monoclonal surfactant protein D (SP-D) antibody (cat. no. sc-59695; Santa Cruz; diluted 1:500), or rabbit monoclonal anti- $\beta$-actin (cat. no. sc-130656; Santa Cruz; diluted 1:2,500) the membrane was washed three times. The membranes were then incubated with the required goat anti-mouse or goat anti-rabbit secondary antibody (Santa Cruz; diluted 1:2,500) for $1 \mathrm{~h}$. After being washed three times with TBST buffer, 
Table II. Upregulated expression genes comparison BCGi group with BCG group.

\begin{tabular}{llccc}
\hline Symbol & Gene ID & $\begin{array}{c}\text { Relative level of gene } \\
\text { expression in group BCGi }\end{array}$ & $\begin{array}{c}\text { Relative level of gene } \\
\text { expression in group BCG }\end{array}$ & $\begin{array}{c}\text { Gene expression ratio of } \\
\text { group BCGi to BCG }\end{array}$ \\
\hline Igh-6 & XM_177464 & 1.827041 & 0.801591 & 2.3 \\
Lbp & NM_008489 & 1.180695 & 0.512301 & 2.3 \\
Ltf & NM_008522 & 0.772659 & 0.343613 & 2.2 \\
Ly96 & NM_016923 & 1.061771 & 0.492027 & 2.2 \\
Ncf4 & NM_008677 & 2.166263 & 0.446541 & 4.9 \\
Nfkb1 & NM_008689 & 7.759150 & 2.590354 & 3.0 \\
Nfkb2 & NM_019408 & 5.276989 & 1.089581 & 4.8 \\
Nfkbia & NM_010907 & 7.424547 & 3.152819 & 2.4 \\
Nos2 & NM_010927 & 2.641727 & 0.794053 & 3.3 \\
Prg2 & NM_008920 & 1.436093 & 0.340494 & 4.2 \\
Sftpd & NM_009160 & 4.856252 & 0.517239 & 9.4 \\
Stab1 & NM_138672 & 1.390140 & 0.485789 & 2.9 \\
Tlr4 & NM_021297 & 1.866528 & 0.528936 & 3.5
\end{tabular}

BCG, Bacille Calmette-Guérin; BCGi, BCG with intracellular pathogen resistance I.

Table III. Downregulated genes comparison BCGi group with BCG group.

\begin{tabular}{llccc}
\hline Symbol & Gene ID & $\begin{array}{c}\text { Relative level of gene } \\
\text { expression in group BCGi }\end{array}$ & $\begin{array}{c}\text { Relative level of gene } \\
\text { expression in group BCG }\end{array}$ & $\begin{array}{c}\text { Gene expression ratio of } \\
\text { group BCGi to BCG }\end{array}$ \\
\hline Cd14 & NM_009841 & 0.657430 & 1.817355 & 0.36 \\
Cdldl & NM_007639 & 0.237848 & 0.523217 & 0.45 \\
Chuk & NM_007700 & 0.626025 & 2.156289 & 0.29 \\
Csf3 & NM_009971 & 0.565524 & 1.676739 & 0.34 \\
Ppbp & NM_023785 & 0.725782 & 2.579958 & 0.28 \\
Cxcr4 & NM_009911 & 0.822538 & 4.387695 & 0.19 \\
Plunc & NM_011126 & 1.071701 & 5.285196 & 0.20 \\
\hline
\end{tabular}

BCG, Bacille Calmette-Guérin; BCGi, BCG with intracellular pathogen resistance I.

signals were detected using the DAB Horseradish Peroxidase Color Development kit (Beyotime Institute of Biotechnology).

Reverse transcription-quantitative PCR (RT-qPCR). Total RNA was extracted by DNA-free RNA mini extraction kit (Watson, Shanghai, China). Total RNA (1 $\mu \mathrm{g})$ was used for cDNA synthesis, which was conducted by reverse transcription using the PrimeScript RT reagent Kit Perfect Real time (Takara Biotechnology Co., Ltd.). Relative quantification was performed using SYBR-Green assays (Roche Diagnostics $\mathrm{GmbH}$, Mannheim, Germany) for the target genes (mRNA of immune related genes), with glyceraldehyde 3-phosphate dehydrogenase (gapdh) mRNA as an endogenous control. The primers were synthetized at Invitrogen and Applied Biosystems (Thermo Fisher Scientific, Inc., Waltham, MA, USA) and are displayed in Table I. The cycling parameters were as follows: Initial denaturation at $95^{\circ} \mathrm{C}$ for $5 \mathrm{~min}$; 40 cycles of denaturation at $95^{\circ} \mathrm{C}$ for $10 \mathrm{sec}$, annealing at $59^{\circ} \mathrm{C}$ for $15 \mathrm{sec}$, and extension at $72^{\circ} \mathrm{C}$ for $20 \mathrm{sec} ; 85^{\circ} \mathrm{C}$ for $5 \mathrm{sec}$ to obtain fluorescence signals with a final extension at $72^{\circ} \mathrm{C}$ for 5 min. The thermocycler was an Applied Biosystems 7300 Quantitative PCR instrument (Applied Biosystems). The dates were analyzed by 7300 Real Time fluorescence quantitative PCR analysis system. Expression values of target genes were calculated using the $2^{-\Delta \Delta \mathrm{Cq}}$ method (7).

Oligo microarray of immune-related genes from murine lung tissue. Total RNA was extracted from 5 individual lung tissues from each group with TRIzol (Takara Biotechnology Co., Ltd.). The total RNA was used for cDNA synthesis and biotin-labeled cRNA linear synthesis using the TrueLabeling-AMP Linear RNA Amplification kit (Superarray Bioscience; Qiagen, Hilden, Germany). Expression profiles of mouse immune responses were determined at KangChen Corporation (Shanghai, China) using the Oligo Innate \& Adaptive Immune Responses Microarray which contained 113 probes of immune-related genes from mice. The raw data was analyzed by the GE Array Expression Analysis Suite (GE Healthcare). The level of cDNA was determined to be differentially expressed when there was a $>2$-fold change compared with control, and it was identified 

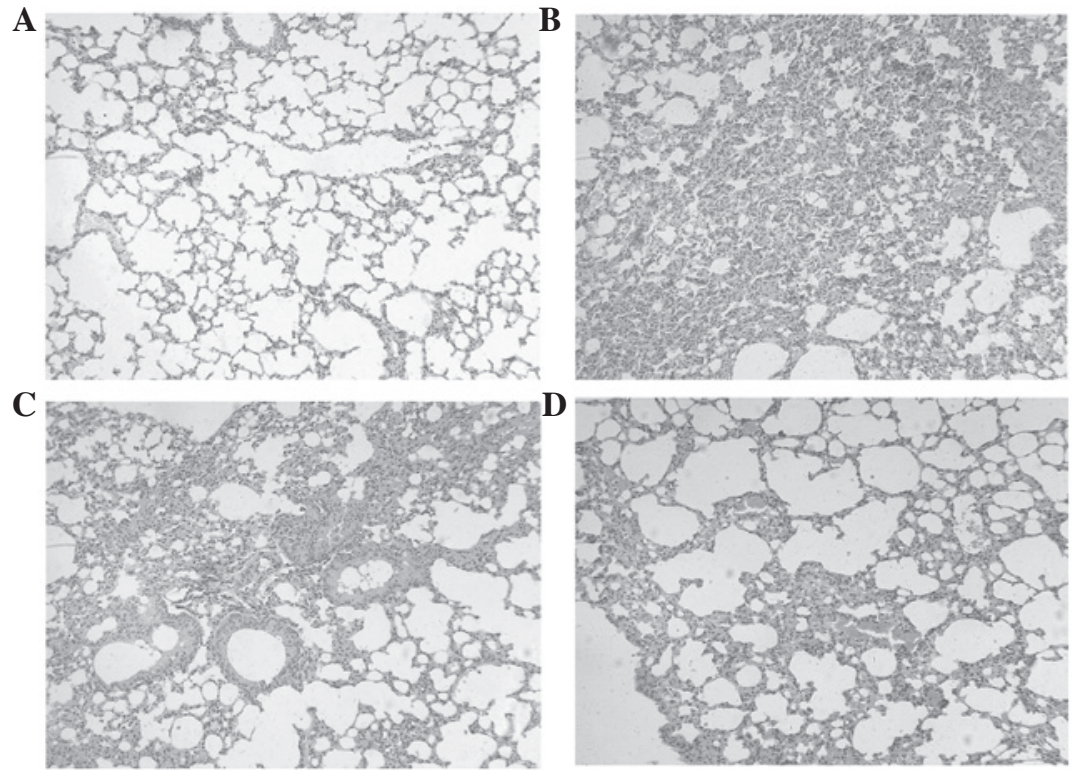

Figure 4. Histology of the lungs of $\mathrm{C} 3 \mathrm{HeB} / \mathrm{FeJ}$ mice after 11 weeks of treatment. The $\mathrm{C} 3 \mathrm{HeB} / \mathrm{FeJ}$ mice were vaccinated with PBS, BCG or BCGi 7 times at 3-day intervals. The mice were then intranasally challenged with live H37Rv at 4 weeks after the last vaccination. The mice were sacrificed 4 weeks after H37Rv infection. (A) Control group, mice were vaccinated with PBS but not infected with H37Rv. (B) PBS group, mice infected with H37Rv after vaccination with PBS. (C) BCG group, mice infected with H37Rv after vaccination with BCG. (D) BCGi group, mice infected with H37Rv after vaccination with BCGi. Stain, hematoxylin and eosin; magnification, x200. BCG, Bacille Calmette-Guérin; BCGi, BCG with intracellular pathogen resistance I; PBS, phosphate-buffered saline.
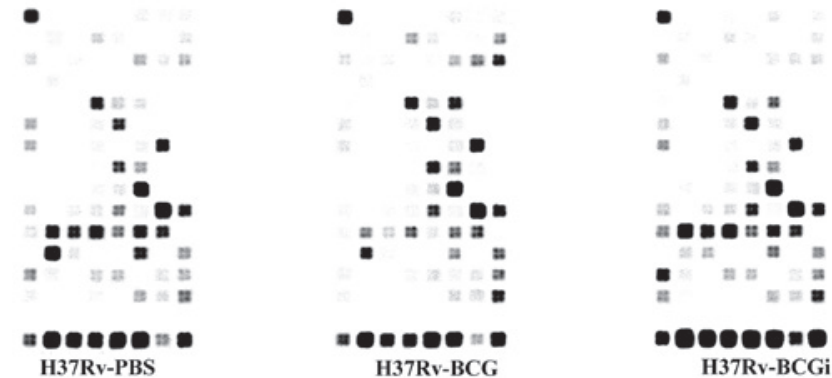

Figure 5. Expression of immune-related genes from murine lung tissues in the BCG group and BCGi group. X-film results of oligo microarray of 113 immune-related genes. H37Rv-PBS, mice infected with H37Rv after vaccination with PBS; H37Rv-BCG, mice infected with H37Rv after vaccination with BCG; H37Rv-BCGi, mice infected with $\mathrm{H} 37 \mathrm{Rv}$ after vaccination with BCGi. BCG, Bacille Calmette-Guérin; BCGi, BCG with intracellular pathogen resistance I; PBS, phosphate-buffered saline.

as significantly changed using the using the Rotor-gene 6.0 Microarrays Analysis method with false discovery rate $<0.05$.

Statistical analysis. All data are expressed as the mean \pm standard deviation. Differences between groups were assessed by one way analysis of variance with a Bonferroni post hoc test. $\mathrm{P} \leq 0.05$ differences were considered to indicate a statistically significant difference. All analyses were performed using the SPSS statistical software for Windows, version 10.1.4 (SPSS Inc., Chicago, IL, USA).

\section{Results}

Mice are intranasally infected with H37Rv. Live H37Rv was intranasally delivered to mice. Lung counts were conducted 3 days after infection to ensure the mice were infected with
TB. As shown in Fig. 1A, the H37Rv (which has an arrow was observed in the lung tissues by acid fast staining. A total of $200 \mu \mathrm{l}$ of the homogenate of lung and spleen tissue $(10 \mathrm{~g} / 100 \mathrm{ml})$ was added to each of the plates containing Löwenstein-Jensen medium. Subsequently, these were incubated for 4 weeks, and then the bacterial colonies were observed in the medium. Bacterial counts in the organs were shown and counted in Löwenstein-Jensen medium following the incubation period (Fig. 1B). H37Rv was not identified in the control mice.

BCGi was successfully delivered to mice intranasally. Iprl mRNA in the lung and spleen tissues was observed in the BCGi group by RT-PCR (Fig. 2A). Iprl expression in the lung and spleen tissues in BCGi was also conformed by western blot analysis (Fig. 2B). This result indicated that the recombinant vaccine BCGi was successfully intranasally delivered to mice. Furthermore, BCG was shown to be effective in delivering Ipr1 to target tissues and resulting in its expression.

Coefficient of lung and spleen in BCGi group were lower than that of the BCG group. Compared with the phosphate-buffered saline (PBS) group, the organ coefficients in the BCGi group were significantly decreased $(\mathrm{P}<0.01)$, while the differences between the PBS and BCG groups was not significant $(\mathrm{P}>0.05)$. Conversely, the lung and spleen coefficients in the BCGi group were lower than that of the $\mathrm{BCG}$ group $(\mathrm{P}<0.01$; Fig. $3 \mathrm{~A}$ and $\mathrm{B})$.

Decrease in bacterial load in the lung and spleen tissues of infected mice following vaccination with BCGi. The bacterial loads were measured by placing serial dilutions onto Löwenstein-Jensen media. As shown in Fig. 3C and D, the BCGi group had significantly fewer $\mathrm{cfu} / \mathrm{g}$ in the lungs and spleen than the PBS and BCG groups $(\mathrm{P}<0.01)$. Although the 


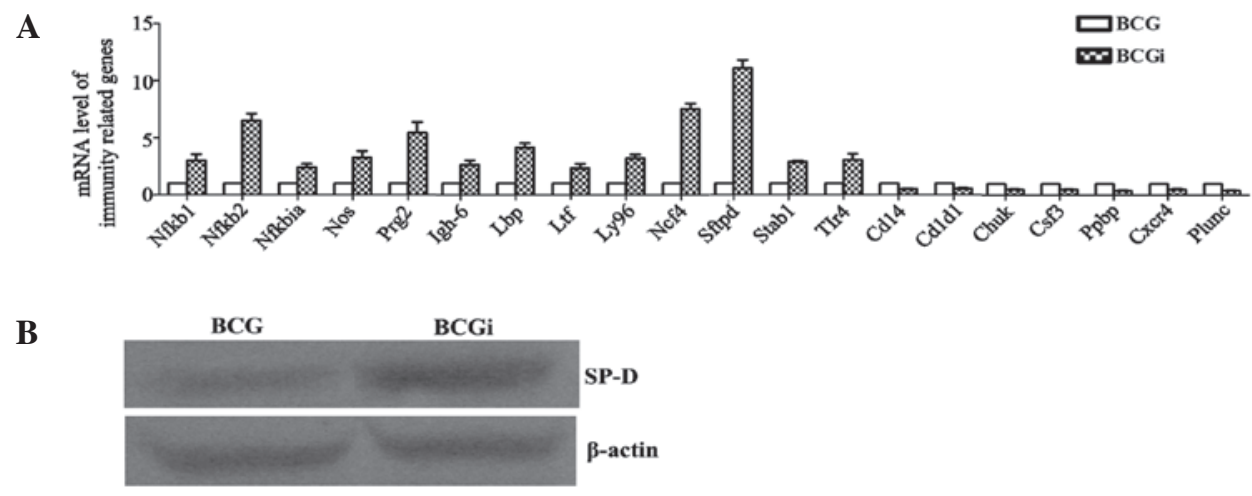

Figure 6. Quantity real-time PCR and western-blot assay evaluated the 20 differential expression immune related genes in BCG group and BCGi group. (A) MRNA level of immune related genes between BCG group and BCGi group by quantity real-time PCR. (B) SP-D expression in lung tissues was examined by western-blot analysis.

BCG group had fewer cfu/g in organs than the PBS group, the difference was not significant.

Histology result. Different treatments resulted in different pathological changes of the lung tissues. Subsequent to the primary vaccination (11 weeks), the lesions of the lung tissues in the PBS group (vaccinated with PBS) were excessive and extensive, and the normal alveolar structure had disappeared (Fig. 4A). The lesions in the lung tissues in the BCG group (vaccinated with $\mathrm{BCG}$ ) and $\mathrm{BCGi}$ group (vaccinated with $\mathrm{BCGi}$ ) were decreased compared with the PBS group (Fig. 4B-D). Abundant foamy cytoplasm and cholesterol crystallization were observed in the BCG group and BCGi group. Compared with the BCG group, the lesions of the lung tissues in the BCGi group were fewer and less severe, and the alveoli wall structure was more defined.

Overexpression of SD-P may be responsible for the stronger immunity of BCGi against tuberculosis than BCG. Expression profiles of mouse immune responses were determined using oligo microarray which contained 113 probes of immune-related genes from mice (Fig. 5). The result showed that there were 20 differentially expressed genes with a $>2$-fold change compared with the BCG group. Specifically, there were 13 upregulated genes: Igh6, Lbp, Ltf, Ly96, Ncf4, Nfkb1, Nfkb2, Nfkbia, Nos2, Prg2, Sftpd, Stab1 and Tlr4 (Table II); and 7 downregulated genes: $C d 14, C d l d l$, Chuk, Csf 3, Ppbp, Cxcr4 and Plunc in the BCGi group compared with the BCG group (Table III). The expression level of these 20 genes was further determined by RT-qPCR (Fig. 6A), which showed the same trend as the microarray. In particular, the expression level of $S f t b p$, an immune-related gene, increased $>10$-fold in BCGi group compared with the BCG group. SP-D was encoded by the Sftbp gene and its expression level in the BCGi group was significantly higher than that of the BCG group as determined by western blot analysis (Fig. 6B).

\section{Discussion}

Tuberculosis is a major challenge to global public health. However, research has indicated that only $10 \%$ of individuals infected with M.tuberculosis will develop disease (8) and there are factors that determine the susceptibility in individuals (9). Association studies have identified various host genetic factors that affect susceptibility to TB. The mouse gene Iprl within the sst1 locus has been shown to contribute to innate immunity in a murine model of M.tuberculosis infection (5). The human homologue of Iprl is SP110, which may be a promising candidate for controlling $M$. tuberculosis infections. The genotypes and haplotypes of $S P 110$ have been widely investigated in recent years, yet the results were somewhat contradictory and indeterminate $(10,11)$. In 2011, Liang et al (12) demonstrated that the genotypes and haplotypes of SP110 may be associated with susceptibility to TB in the Chinese population. Previous studies indicated that Ipr1 could enhance the effect of macrophages against intracellular pathogens, such as mycobacterium (13). Macrophages are key in innate immunity; however, the intrinsic link between this gene and macrophages or the innate immune system was unclear. Thus, the present study investigated the underlying mechanism using IPR1 to transform BCG and determined its effect on M. tuberculosis infection.

In the present study, BCG was altered and carried the IprI gene into macrophages in vivo. $\mathrm{C} 3 \mathrm{HeB} / \mathrm{FeJ}$ mice were selected as the test animal as they do not contain the Iprl gene in its genome and are sensitive to $M$. tuberculosis. The immune effect of this recombinant vaccine BCGi was evaluated in the mice by determining the organ coefficient, bacterial load and the histopathological characterization of the infected organs. It was shown that the bacterial load in the lung and spleen was significantly reduced in the BCGi group compared with the PBS and BCG groups. Bacterial load was shown to be an important indicator of immune efficacy against $M$. tuberculosis infection in animal studies (14). Also known as the organ coefficient is the ratio of the weight of the organs and the experimental animals. The normal organ coefficient is relatively constant. However, an increase in the organ coefficient is suggestive of organ congestion, edema, or hypertrophy and therefore may reflect the intensity of infection and inflammation to a certain extent. The coefficient of the lung and spleen in the BCGi group were lower than those in the BCG group and PBS group, and closer to the state of the mice without infection. This result indicated that BCGi as a vaccine resulted in a greater reduction in bacterium infection and the inflammatory response compared with BGC. Histopathological 
results showed that the lesions in the lungs of the BCG group less severe slighter than those in the PBS group, but more advanced than in the BCGi group. These results confirmed that the recombinant vaccine $\mathrm{BCGi}$ had a stronger protective effect against $M$. tuberculosis than BCG.

Studies have shown that the TLR signaling pathway is important in resistance to $M$. tuberculosis infection by macrophages. TLR1, TLR6, TLR2, TLR4, TRIF, Irak, Traf6, Myd88 and NF- $\kappa \mathrm{B}$ are involved in the TLR signaling pathway (15). Certain surface structures of $M$.tuberculosis are able to combine with the ligands of TLR2 and TLR4, such as LAM (lipoarabinomannan) a $19 \mathrm{kD}$ lipoprotein, and the $38 \mathrm{kD}$ sugar lipoprotein, HSP70 and so on (16). After TLR2 or TLR4 bind to the corresponding ligands, NF- $\kappa \mathrm{B}$ is activated by the MyD88-dependent and non-dependent pathways, which caused the release of a variety of inflammatory cytokines and upregulation of the expression of CD80, CD86 and other co-stimulatory molecules on the surface of antigen-presenting cells. These changes improve the innate immune ability to eliminate the pathogens at an early stage (17). The result of oligo microarray showed that the gene expression of TLR4 increased $>3$ times in the BCGi group compared with the BCG group, while no change in the levels of TLR2, Irak, Traf6, Myd88 was identified. It indicated that Ipr1 enhancing the innate immune against $M$.tuberculosis may occur through the Myd88-independent pathway of TLR4. In addition, TLRs signaling pathway could also activate other mechanisms against $M$. tuberculosis infection, including apoptosis and NO production. In this study, the expression of Nos 2 was notably increased in BCGi group. This suggested that Iprl may promote the production of NO by macrophages through the TLR signaling pathway, and NO was able to damage the structure of M.tuberculosis so that the bacteria may be eliminated. In total, 113 immune-related genes from mice were detected by oligo microarray, however, only 20 of these genes showed $>2$-fold change in expression compared with the BCG group. Notably, among these 20 genes, stfpd exhibited the greatest change in expression ( $>10$-fold) in the BCGi group, compared with the BCG group. SP-D was encoded by the stfpd gene, is a component of the lung innate immunity that enhances clearance of pathogens and modulates inflammatory responses (18). The lung is continuously exposed to inhaled pollutants, microbes and allergens. Thus, the pulmonary immune system has to defend against harmful pathogens, but not produce an inappropriate inflammatory response to harmless particles. In the bronchoalveolar space this critical balance is maintained by innate immune proteins, termed surfactant proteins, such as surfactant SP-D, which is central to the pulmonary host defense. SP-D is an epithelial-cell-derived immune modulator that belongs to the small family of structurally related $\mathrm{Ca}\left({ }^{2+}\right)$-dependent $\mathrm{C}$-type collagen-like lectins. Although collectins can be detected on mucosal surfaces of various organs, SP-D is constitutively expressed in the lung at high concentrations (19). Research has demonstrated that SP-D binds carbohydrates, lipids, lipoteichoic acid and nucleic acids with a broad spectrum specificity and initiates phagocytosis of inhaled pathogens as well as apoptotic cells (20). Therefore, pathogen binding and aggregation by lung collectins is an method of phagocytic elimination. Early studies showed that SP-A (similar to SP-D) enhanced the phagocytosis of
M. tuberculosis and M. bovis by alveolar macrophages $(21,22)$ However, the details of the innate or adaptive immunity modulation by SP-D are not well understood. Certain studies have demonstrated that SP-D could recognize, bind and modulate the function of CD14 (23), TLR-2 (24) and TLR4 (25). Lack of SP-D in gene-deficient mice resulted in a failure to increase TLR4 expression during allergic inflammation in comparison with wild-type (26). In addition, SP-D was shown to enhance the amount of oxygen radicals produced by alveolar macrophages and neutrophils (27).

In conclusion, the Iprl modified BCG has stronger immunity than BCG against tuberculosis infection in mice, and could be a candidate vaccine against $M$. tuberculosis. Ipr1 was shown to activate the TLR4 signaling pathway to increase inflammatory cytokine release, co-stimulatory molecule expression on the surface of APCs, apoptosis and NO production by macrophages. Conversely, Ipr1 could improve SP-D expression in lung tissues, which could bind and initiate the phagocytosis of pathogens.

\section{Acknowledgements}

This study was supported by grants from the National Science Foundation of China (grant no. 30901280) and Chongqing Municipal Commission of Health and Family Planning (2015MSXM081).

\section{References}

1. Global tuberculosis control: surveillance, planning, financing. WHO report 2007. Geneva, World Health Organization (WHO/HTM/TB/2007.376).

2. Dos Santos JL, Lima MM, Trindade AB, Carnavalli F, Melchior AC and Chin CM: Tuberculosis: Challenges to improve the treatment. Curr Clin Pharmacol, 2013. (Epub ahead of print).

3. Montagnani C, Chiappini E, Galli L and de Martino M: Vaccine against tuberculosis: What's new? BMC Infect Dis 14 (Suppl 1): S2, 2014.

4. Martin C: Tuberculosis vaccines: Past, present and future. Curr Opin Pulm Med 12: 186-191, 2006.

5. Pan H, Yan BS, Rojas M, Shebzukhov YV, Zhou H, Kobzik L, Higgins DE, Daly MJ, Bloom BR and Kramnik I: Ipr1 gene mediates innate immunity to tuberculosis. Nature 434: 767-772, 2005.

6. Wang YW, Xu L, Zhang L, He YL, Yang $\mathrm{C}$ and Huang AL: Construction the recombinant BCG targeting delivering IprI into macrophages: A new strategy of vaccine against tuberculosis. African Journal of Microbiology Research 7: 533-540, 2013.

7. Livak KJ and Schmittgen TD: Analysis of relative gene expression data using real-time quantitative PCR and the 2(-Delta Delta C(T)) Method. Methods 25: 402-408, 2001.

8. Fang R, Li X, Li J, Wu J, Shen X, Gui X, DeRiemer K, Liu L, Mei J and Gao Q: Mixed infections of Mycobacterium tuberculosis in tuberculosis patients in Shanghai, China. Tuberculosis (Edinb) 88: 469-73, 2008.

9. Bellamy R: Genetic susceptibility to tuberculosis. Clin Chest Med 26: 233-246, 2005

10. Png E, Alisjahbana B, Sahiratmadja E, Marzuki S, Nelwan R, Adnan I, van de Vosse E, Hibberd M, van Crevel R, Ottenhoff TH and Seielstad M: Polymorphisms in SP110 are not associated with pulmonary tuberculosis in Indonesians. Infect Genet Evol 12: 1319-1323, 2012.

11. Lei X, Zhu H, Zha L and Wang Y: SP110 gene polymorphisms and tuberculosis susceptibility: A systematic review and meta-analysis based on 10624 subjects. Infect Genet Evol 12: 1473-1480, 2012.

12. Liang L, Zhao YL, Yue J, Liu JF, Han M, Wang H and Xiao H: Association of SP110 gene polymorphisms with susceptibility to tuberculosis in a Chinese population. Infect Genet Evol 11: 934-939, 2011. 
13. Kramnik I: Genetic dissection of host resistance to Mycobacterium tuberculosis: The sst1 locus and the Ipr1 gene. Curr Top Microbiol Immunol 321: 123-148, 2008.

14. Orme IM, McMurray DN and Belisle JT: Tuberculosis vaccine development: Recent progress. Trends Microbiol 9: 115-118, 2001.

15. Quesniaux V, Fremond C, Jacobs M, Parida S, Nicolle D, Yeremeev V, Bihl F, Erard F, Botha T, Drennan M, et al: Toll-like receptor pathways in the immune responses to mycobacteria. Microbes Infect 6: 946-959, 2004.

16. Jung SB, Yang CS, Lee JS, Shin AR, Jung SS, Son JW, Harding CV, Kim HJ, Park JK, Paik TH, et al: The mycobacterial 38-kilodalton glycolipoprotein antigen activates the mitogen-activated protein kinase pathway and release of proinflammatory cytokines through Toll-like receptors 2 and 4 in human monocytes. Infect Immun 74: 2686-2696, 2006.

17. Ospelt $\mathrm{C}$ and Gay S: TLRs and chronic inflammation. Int $\mathbf{J}$ Biochem Cell Biol 42: 495-505, 2010.

18. Hartl D and Griese M: Surfactant protein D in human lung diseases. Eur J Clin Invest 36: 423-435, 2006.

19. Haczku A: Protective role of the lung collectins surfactant protein A and surfactant protein D in airway inflammation. J Allergy Clin Immunol 122: 861-879, 2008.

20. Forbes LR and Haczku A: SP-D and regulation of the pulmonary innate immune system in allergic airway changes. Clin Exp Allergy 40: 547-562, 2010.

21. Gaynor CD, McCormack FX, Voelker DR, McGowan SE and Schlesinger LS: Pulmonary surfactant protein A mediates enhanced phagocytosis of Mycobacterium tuberculosis by a direct interaction with human macrophages. J Immunol 155: 5343-5351, 1995.
22. Weikert LF, Edwards K, Chroneos ZC, Hager C, Hoffman L and Shepherd VL: SP-A enhances uptake of bacillus Calmette-Guerin by macrophages through a specific SP-A receptor. Am J Physiol 272: L989-L995, 1997.

23. Chiba H, Sano H, Iwaki D, Murakami S, Mitsuzawa H, Takahashi T, Konishi M, Takahashi $\mathrm{H}$ and Kuroki Y: Rat mannose-binding protein a binds CD14. Infect Immun 69: $1587-1592,2001$

24. Ohya M, Nishitani C, Sano H, Yamada C, Mitsuzawa H, Shimizu T, Saito T, Smith K, Crouch E and Kuroki Y: Human pulmonary surfactant protein D binds the extracellular domains of Toll-like receptors 2 and 4 through the carbohydrate recognition domain by a mechanism different from its binding to phosphatidylinositol and lipopolysaccharide. Biochemistry 45: 8657-8664, 2006.

25. Guillot L, Balloy V, McCormack FX, Golenbock DT, Chignard M and Si-Tahar M: Cutting edge: The immunostimulatory activity of the lung surfactant protein-A involves toll-like receptor 4. J Immunol 168: 5989-5992, 2002.

26. Zhang L, Ikegami M, Crouch EC, Korfhagen TR and Whitsett JA: Activity of pulmonary surfactant protein-D (SP-D) in vivo is dependent on oligomeric structure. J Biol Chem 276: 19214-19219, 2001

27. van Iwaarden JF, Shimizu H, Van Golde PH, Voelker DR and Van Golde LM: Rat surfactant protein D enhances the production of oxygen radicals by rat alveolar macrophages. Biochem J 286: $5-8,1992$. 